\title{
A CITYGML EXTENSION FOR HANDLING VERY LARGE TINS
}

\author{
K. Kumar*, H. Ledoux, J. Stoter
}

3D Geoinformation, Delft University of Technology, The Netherlands - (k.kavisha, h.ledoux, j.e.stoter)@ tudelft.nl

KEY WORDS: CityGML, GML, Terrains, TINs.

\begin{abstract}
:
In addition to buildings, the terrain forms an important part of a 3D city model. Although in GIS terrains are usually represented with 2D grids, TINs are also increasingly being used in practice. One example is 3DTOP10NL, the 3D city model covering the whole of the Netherlands, which stores the relief with a constrained TIN containing more than 1 billion triangles. Due to the massive size of such datasets, the main problem that arises is: how to efficiently store and maintain them? While CityGML supports the storage of TINs, we argue in this paper that the current solution is not adequate. For instance, the 1 billion+ triangles of 3DTOP10NL require 686 GB of storage space with CityGML. Furthermore, the current solution does not store the topological relationships of the triangles, and also there are no clear mechanisms to handle several LODs. We propose in this paper a CityGML extension for the compact representation of terrains. We describe our abstract and implementation specifications (modelled in UML), and our prototype implementation to convert TINs to our CityGML structure. It increases the topological relationships that are explicitly represented, and allows us to compress up to a factor of $\sim 25$ in our experiments with massive real-world terrains (more than 1 billion triangles).
\end{abstract}

\section{INTRODUCTION}

A 3D city model is a digital representation of the geographical objects within a city (Stadler and Kolbe, 2007). At first, 3D city models were mainly used for visualization, but with the advancements in geoinformation technologies, they have gained importance in different domains like urban planning, 3D cadastre, environmental simulations, etc.; see Biljecki et al. (2015) for an overview. It should be noticed that, so far, the applications of 3D city modelling are mostly centred around the buildings; other features, e.g. terrain/relief, vegetation, roads, water bodies, bridges, are often ignored. We focus in this paper on the terrain part of a 3D city model, and particularly on its storage as a TIN (Triangulated Irregular Network), which is, apart from grids, more and more used in practice. TINs are a network of non-overlapping triangles formed by the interconnection of irregularly spaced points (Kumler. 1994). They are generally constructed using Delaunay triangulation but this is not always true (Rippa, 1990, Dyn et al. 1990). Everytime the typical inputs for TIN construction are not only a set of vertices but can include other constraints like segments or polygons, etc.In such cases, a constrained triangulation can be constructed. A CDT (Constrained Delaunay Triangulation) is like DT however every given input segment appears as an edge in the triangulation (Shewchuk 1996).

While the international standard CityGML (City Geography Markup Language) allows us to store terrains as TINs (with and without constraints) (OGC 2012), we argue in this paper that the current solution is not suitable to store massive TINs. Firstly, with massive TINs, the datasets become very large, which greatly hinders exchange and dissemination. Secondly, there is very little topological information stored, which prevents us from using the triangles for analysis. The cause of these two problems are that triangles are stored with the OGC Simple Feature structure, which stores independently each triangle, and moreover repeats several vertices. We discuss in Section 3 the other limitations of the current solutions. As an example, consider 3DTOP10NL, the 3D city model of Netherlands (Kadaster 2015), which covers the whole country, including buildings, terrain, roads, canals, etc. (see Figure 1). It is constructed by adding the third dimen-

\footnotetext{
${ }^{*}$ Corresponding author
}

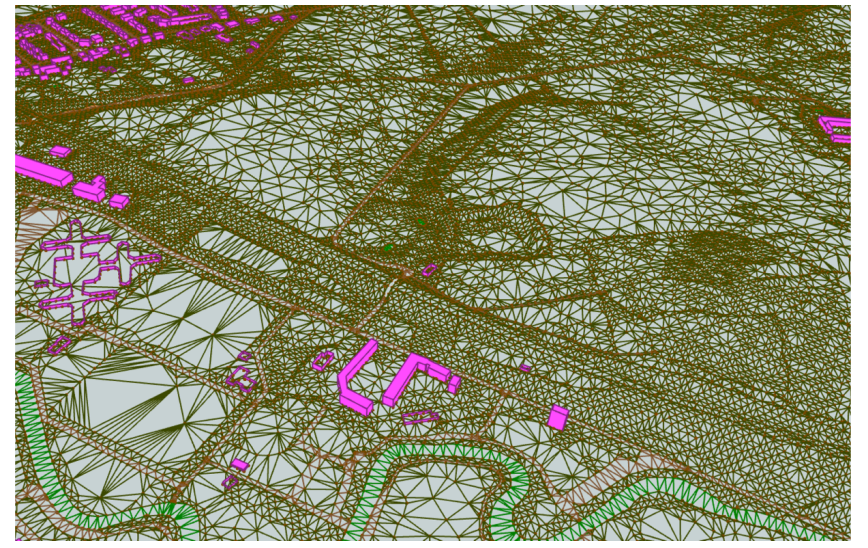

Figure 1: An area of the 3DTOP10NL dataset. Notice that the terrain, the roads, the water courses have all been triangulated, forming one large triangulation for the whole of the Netherlands.

sion from a point cloud, obtained from airborne laser-scanners, to the objects in the 2D topographic map TOP10NL (Elberink et al. 2013). Its terrain is a constrained TIN with more than 1 billion triangles $(1,156,641,666$ to be exact). Storing it with the current solution of CityGML requires around $686 \mathrm{~GB}$ of storage space. One can imagine that if all the elevation points from the point cloud had been used (around 640 billions, thus around 1.3 trillion triangles), then the file size would clearly prevents us from using the dataset.

In this paper, we propose a CityGML extension (in the form of an ADE (Application Domain Extension) for the compact representation of TINs. Instead of repeating several times each coordinates of the triangles, we use an index of vertices and triangles are represented as triples of indices. We describe in our proposed improvements in Section 4 and in Section 5 we discuss the results of the prototype implementation for converting 
TINs to our CityGML extension structure. This allows us to compress up to a factor of $\sim 25$ massive real-world terrains such as the 3DTOP10NL.

\section{TERRAINS IN CITYGMI}

CityGML is an XML based data model for the storage and exchange of virtual 3D city models (Groger et al. 2012). It is implemented as an application schema of GML3 (Geography Markup Language version 3.1.1) and models 3D geometry along with semantics. The data model of CityGML comprises of a core module and several thematic extension modules like Building, Relief, Bridge, LandUse, Tunnel, Vegetation, WaterBody, etc. (Groger et al., 2012). Here we consider the terrain as a DTM (Digital Terrain Model). In practice, the terms DTM and DSM (Digital Surface Model) are often assumed to be synonymous. Before going further, the terms DTM and DSM should be clear. DTM depicts the surface of the earth without any relief objects i.e. the bare earth (shown in blue in Figure 2). DSM depicts the surface of the earth including the relief objects on it (shown in red in Figure 2).

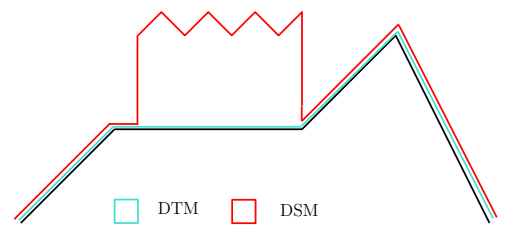

Figure 2: Digital Terrain Model

In CityGML, DTM is provided by the thematic module Relief (Groger et al. 2012). The features of the terrain are represented by the class ReliefFeature. The terrain can be represented either as a TIN (TINReflief), or as a Grid (RasterRelief), or as masspoints (MasspointRelief), or as breaklines (BreaklineRelief) (Figure 4). The corresponding GML3 classes are: gml: RectifiedGridCoverage for grids, gml: MultiCurve for break lines, gml:MultiPoint for mass points and gml:TriangulatedSurface or gml: Tin for TINs (Groger et al. 2012). It is also possible to represent terrain as a combination of different terrain types within a single dataset. For instance, terrain can be modelled by a coarse grid with some areas of detailed TIN (Figure 3) or a TIN with break lines to depict a constrained triangulation, etc. The validity of each terrain type is limited to a certain area defined by the validity_extent_polygon.

A TIN in CityGML is stored as a set of linear rings with gml:Triangle geometry (Figure 5). The class TINRelief describes a terrain as a triangulated surface embedded in 3D space. Its geometry is specified by the GML class gml: TriangulatedSurface, which is composed of triangles explicitly specified as trianglePatches with gml:Triangle geometry (Figure 6). Within its subclass gml: Tin, only the points (with 3D coordinates) are represented, along with optional breaklines (which acts as constraints in the triangulation (Shewchuk 1997)), control points, etc. The support for TINs in GML3 as Linear Rings is consistent with OGC Geometry Abstract Specifications, ISO 19107:2003 Spatial schema and OGC Simple Feature Common Architecture (OGC. 2011). The ISO 19107:2003 Spatial schema specifies the conceptual schema for representing and manipulating TINs in compliance with ISO 19103. These abstract specifications are implemented in ISO 19136 GML. GML is an XML encoding (as per ISO 19118) for the storage and exchange of geoinformation (both spatial and non-spatial attributes of spatial features). The geoinformation is modelled according to the conceptual schemas used in ISO 1900 series.

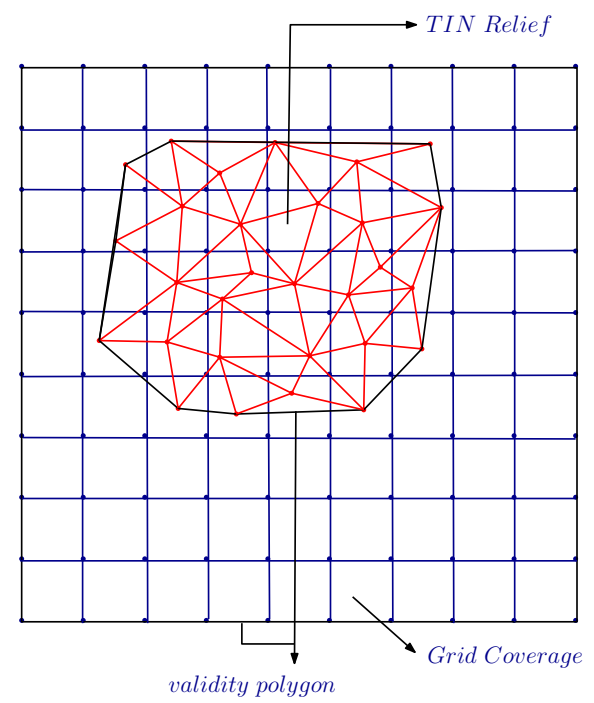

Figure 3: TIN + Grid combination in CityGML. TIN vertices may lie anywhere on the grid and not necessarily at the centre of each grid pixel.

It should be noticed that if only the points of the TIN are stored (and not the triangle), then the triangulation that is calculated with a given triangulator in a downstream application might not always be same. Indeed, the Delaunay triangulation has the property of being unique, but that is only if no four points are co-circular. In practice, this is virtually never the case, especially since many terrain data come from grids where by definition each four points of a cell are co-circular. There exists different algorithms to ensure that a list of points always yield the same Delaunay triangulation: Edelsbrunner and Mücke (1990) use the ordering of the points, and Dyken and Floater (2006) define preferred directions. However, if one stores only their points, there is no guarantee that the algorithm used by the practitioner will have these properties. The problem is made worse when a constrained Delaunay triangulation (CDT) is used, since the algorithms to construct these from points and breaklines are less common and standardised.

\section{PROBLEMS IN STORING MASSIVE TERRAINS IN CITYGML/GML}

CityGML supports the storage of terrains as TINs and Grids. However, there are several problems associated with the storage of massive terrains in CityGML format.

1. Huge data volumes: Since every triangle is stored as a linear ring of vertices (Figure 5), the size of the CityGML files is increased considerably with the repeated storage of vertex information. In a 2D Delaunay triangulation the average degree of a vertex is 6 if the vertices follow a Poisson distribution (Okabe et al. 2009). This suggests that on an average each vertex is stored $6+6 / 3=8$ times thereby, increasing the vertex information redundancy in the CityGML datasets.

2. No topology: There is no storage of topological information of TIN. The triangles are stored individually irrespective of their neighbours. This hinders spatial analysis greatly.

3. No referencing of triangles and their vertices: There is no referencing scheme for the vertices of a triangle. Each triangle is specified with a list of coordinates of its vertices in full which takes more space (Figure 5). There is no indexing of triangles as well. 


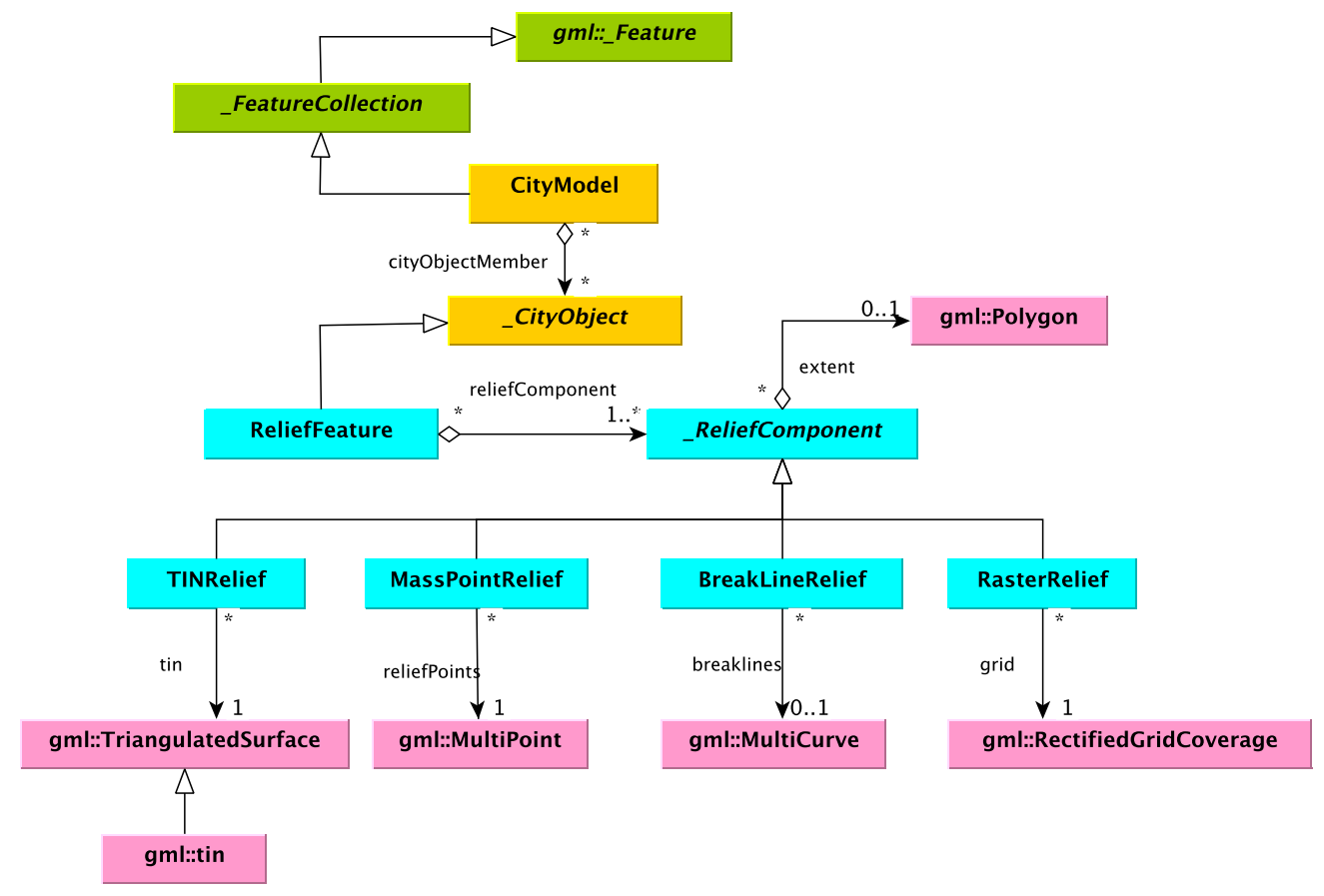

Figure 4: DTM in CityGML (Groger et al., 2012)

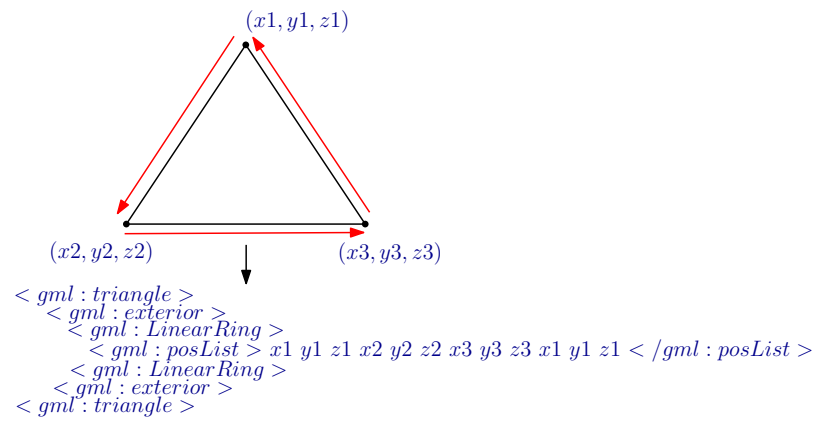

Figure 5: OGC Simple Feature

4. Badly modelled TIN geometry: The class gml:TriangulatedSurf ace comprises of triangle patches forming the TIN. It is defined as a separate subclass of gml:Surface but it could have been defined as a subclass of gml:CompositeSurface. A composite surface requires its elements to be disjoint, not to have overlapping interiors and must be topologically connected along their boundaries. These prerequisites are fulfilled in case of TINs.

5. No specifications for terrain LODs: There is no distinction between different LODs of a terrain in CityGML at geometrical and semantic level. The CityGML 3.0 provides extended LODs for the Building module only and the LOD specifications of other modules like Relief, etc. are left out as unessential features (Löwner et al. 2013, Gröger and Löwner 2016). Giving only a gml: lod does not solve the issue if we cannot identify the difference between LODs.

6. Vertical triangles are not handled: 3DTOP10NL has the skeletons of the urban objects like buildings, roads, etc. integrated in the terrain. In a way, it is not completely a $2.5 \mathrm{D}$ but a $2.8 \mathrm{D}$ model with vertical walls. A $2.8 \mathrm{D}$ models is a 2-manifold surface embedded in a 3D space (Gröger and
Plümer 2005). When a 2.8D model is projected on a 2D surface, the vertical surfaces flatten out which distorts the geometry of the model. There is no mechanism in CityGML to mark out these vertical surfaces so as to remove them while transforming from $3 \mathrm{D}$ to $2 \mathrm{D}$.

7. No support for Tiling: The main memory of a system plays a key role in deciding the maximum size of a dataset that can be processed (Isenburg et al. 2006). If the size of the datasets exceeds the available memory limit then it is split into small parts (called tiles). The concept of tiling the TIN cannot be extended to CityGML as there can be triangles spanning several tiles. Such triangles are repeated in the spanned tiles to complete the OGC SF closed linear ring structure thereby causing information redundancy in the CityGML datasets (Kumar et al. 2016).

8. Unclear TIN and Grid combination : Though CityGML documentation (Groger et al. 2012) describes having a combination of multiple terrain types in a single CityGML instance. But, there can be problems of mismatch of geometry when it comes to combining a TIN and a Grid for a terrain dataset. For instance, in Figure 3 , the terrain is represented by a grid along with a certain area represented by a TIN. The TIN vertices may lie anywhere on the grid and not necessarily at the centre of each grid pixel. Deriving the exact value of elevation of TIN vertices from grid pixels can be an expensive operation in this case.

9. Not suitable for visualization: CityGML is designed for the storage and exchange of 3D city models and not for visualizing them. Visualizing CityGML over web requires to follow another pathway of separating the geometric information from semantic part and transforming it to commonly used 3D graphics formats for visualization. This is owed to the large size and information redundancy in the CityGML datasets. With terrain datasets crossing the billion mark in the count of triangle geometries, the common bottleneck of 


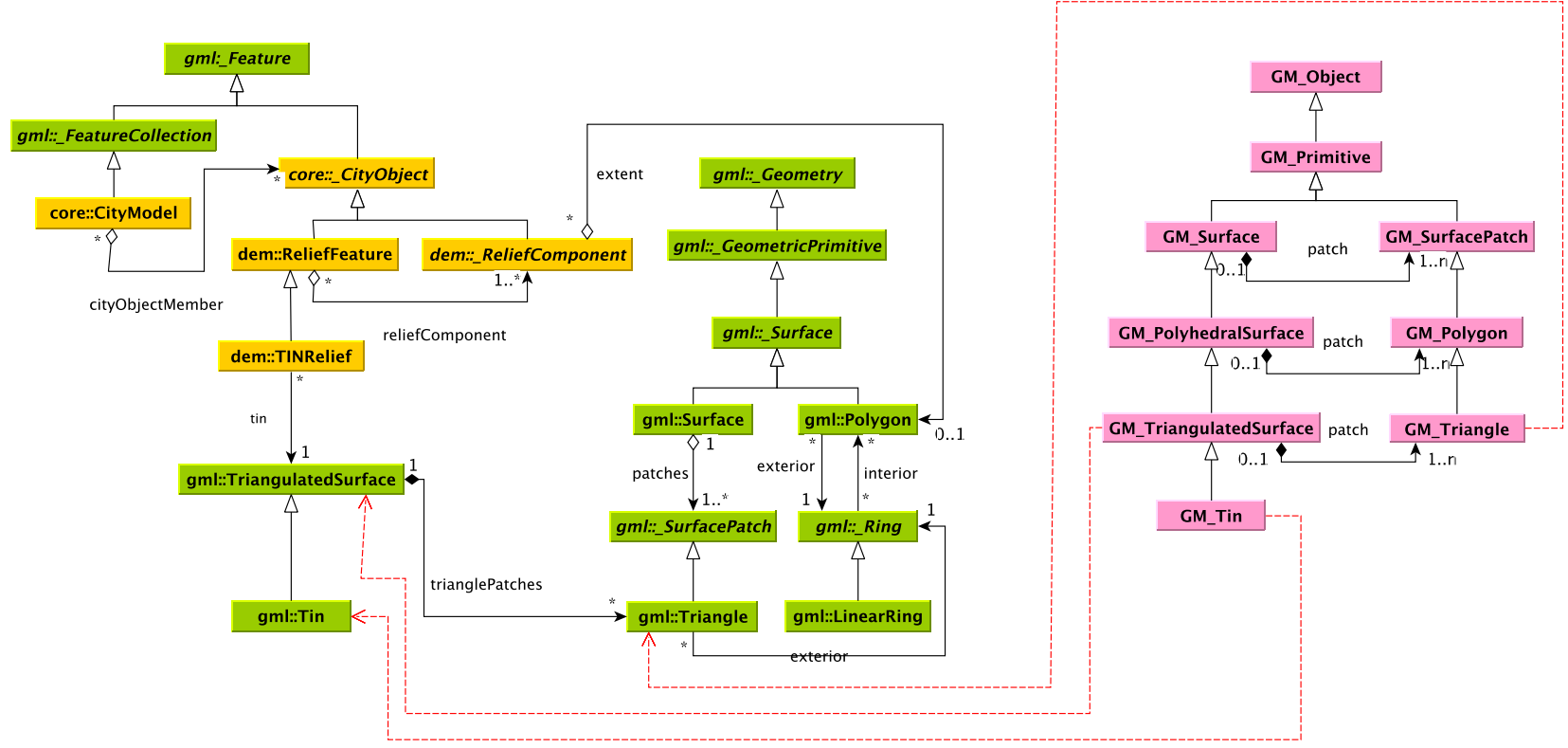

Figure 6: TINs in CityGML and ISO 19107:2003 Spatial schema

web visualization is the rate at which these triangles can be rendered over the graphics engine.

\section{PROPOSED SOLUTION: TERRAINS@CITYGML}

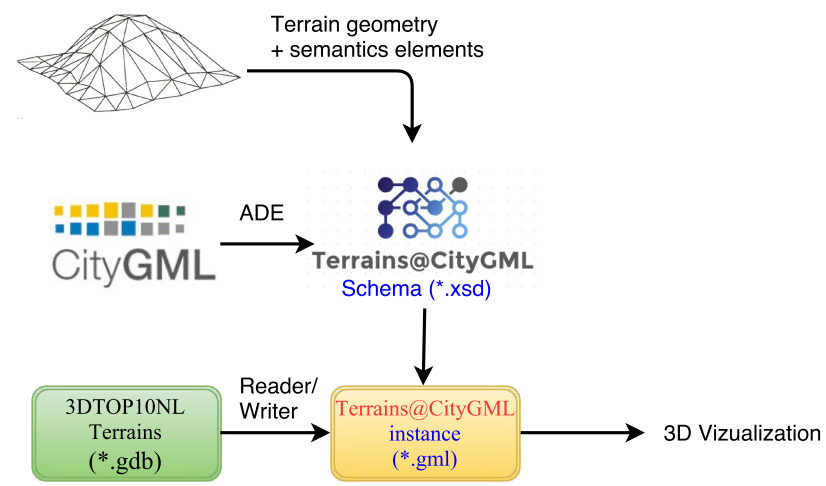

Figure 7: Workflow for proposed solution. CityGML/GML are extented to store terrain geometry and semantics as proposed.

\subsection{Terrains@CityGML}

Based on the weaknesses of the current CityGML/GML schema in storing massive terrains (as highlighted in Section 3), we propose an alternative representation to represent TINs, Terrains $@-$ CityGML. Terrains@CityGML is modelled as an extension to CityGML constructed by adding new geometry encoding schemes for TINs in the CityGML/GML schema. CityGML has the concept of $A D E s$ to extend the schema with new classes/attributes which are not explicitly modelled in CityGML. ADEs are increasingly being used in creating application-specific extensions like for energy modelling (Nouvel et al. 2015), BIM-IFC integration with CityGML (de Laat and Van Berlo 2011), IMGeo for modelling Dutch topographic data in CityGML (Brink et al. 2013), indoor modelling (Kim et al. 2014), noise modelling (Groger et al. 2012), etc. Similarly, there are many specialized versions of GML. For instance, FieldGML is GML based implementation for the representation of geofields in 2D and 3D (Ledoux 2008). Another GML based implementation is $N c M L$ $G M L$ which provides for storing the metadata of netCDF files in GML (Nativi et al. 2005). Others include CSML (Woolf et al. 2006), GeoSciML (Sen and Duffy 2005), etc.

Terrains@CityGML is introduced as an ADE for handling very large TIN representation of terrains in CityGML. The new classes and attributes for terrain semantics and geometry are defined in the schema. The workflow for the proposed solution is depicted in Figure 7 Figure 8 shows the basic class diagram for the proposed Terrains@CityGML. Two new types, TINTerrain and MassPointTerrain for compactly storing the terrains are defined which are capable of storing terrains either as triangles and as a collection of points, respectively. Correspondingly, for the geometry, four new types under the class Terrain_Elements are created: vertices, lines, triangles, source are defined in the GML schema. Terrain_Elements refer to the data elements forming the structure of terrain. They can be:

1. a set of vertices/points in $2 \mathrm{D}$ or $3 \mathrm{D}$ space.

2. a set of connected triangles and their vertices in $2 \mathrm{D}$ or $3 \mathrm{D}$ space.

3. a set of connected triangles, their vertices and additional constraints (breaklines) in 2D or 3D space.

4. a source specifying the data type of the elements.

The $I D s$ of vertices and triangles are stored implicitly and does not require an explicit representation. To represent a constrained triangulation with break lines and masspoints, ConstrainedTINTerrain, a subclass of the TINTerrain is introduced. It inherits its geometry from gml:AbstractGeometryType. The vertices are stored as an array of coordinates of type double and the triangles are stored as an array of vertex indices of type integer (VTP 2012). The generated Terrains@CityGML instances are required to be validated against this schema.

\subsection{Approach for Modelling ADE}

This section presents the approach selected for modelling the CityGML extension. There are different ways of extending CityGML for specific applications. CityGML can be extended with 


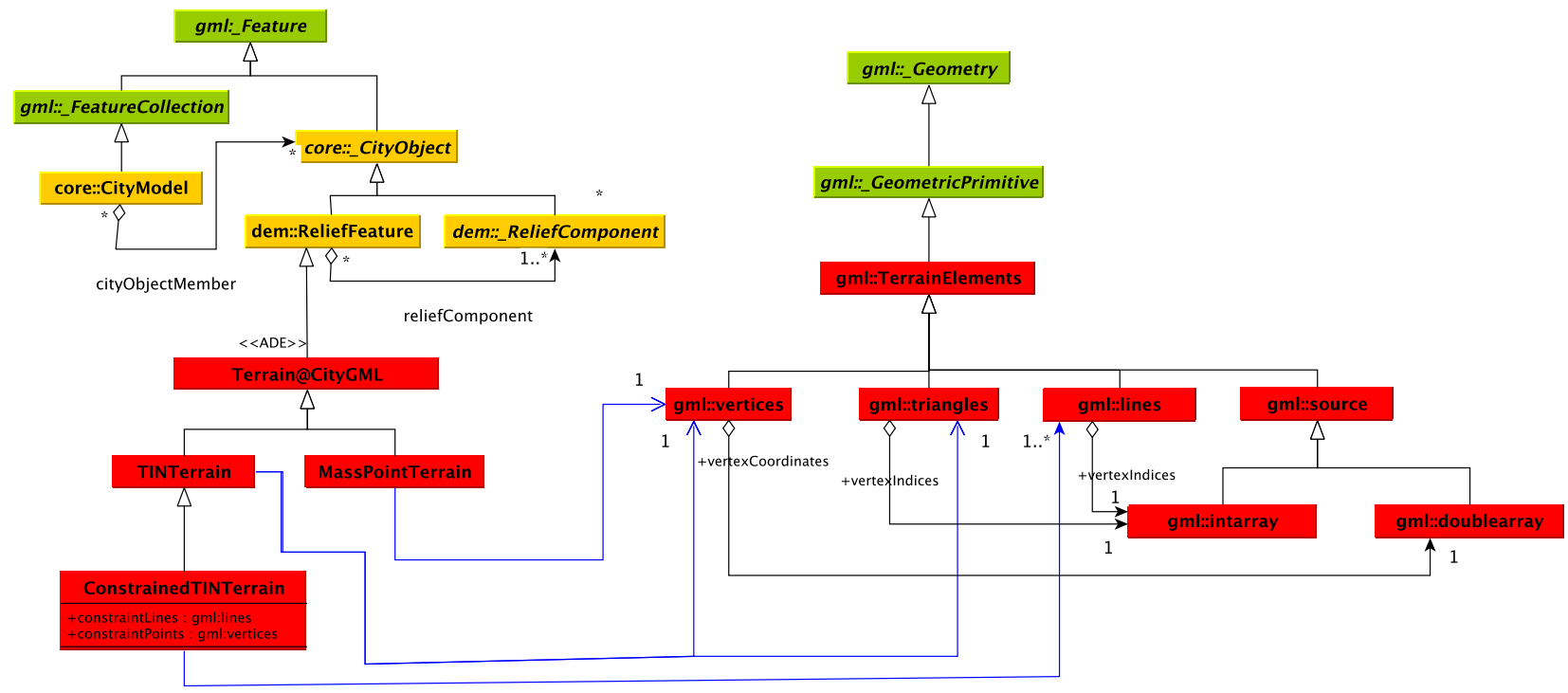

Figure 8: Proposed basic UML of Terrains@CityGML

the introduction of generic attributes and objects in the CityGML files. Any _CityObject can be extended by additional attributes without introducing any change in CityGML schema. The generic extensions of CityGML are provided by the thematic extension module Generics. But they have their own limitations (Groger et al. 2012), namely:

1. There is no formal specification of their names and data types.

2. Such CityGML files cannot be validated against the schema.

3. Name conflicts of the generic attributes and objects may occur.

4. Limited number of data types for generic attributes and objects.

5. Limits semantic and syntactic interoperability.

To ensure semantic and syntactic interoperability for the exchange of application specific information, the structure of information should be represented in a systematic way by defining a separate formal schema which is based on CityGML schema definitions i.e. an ADE. There are different ways to model an ADE in CityGML. They can be modelled directly in the schema or can be generated by first extending the UML (Unified Modelling Language) with application specific information and later exporting the schema. Every CityGML feature type provides a hook in its XML schema definition, that allows to attach additional properties to it by ADEs. This hook is implemented as a GML property of the form _GenericApplicationPropertyOf $<$ Featuretypename $>$ where $<$ Featuretypename $>$ is equal to the name of the feature type definition in which it is included. Another approach for extending CityGML is to add the application specific properties directly in the CityGML classes. These added properties are then marked as an ADE extension with a stereotype. But this suggests editing the standard CityGML schema which is controlled by a different authority: SIG 3D (Special Interest Group 3D). van den Brink et al. (2013) describes six alternatives for modelling ADEs in UML. We adopt the approach of adding application specific properties in the subclass in ADE package. The extension subclass is marked as an ADE extension in the UML model by a stereotype. The CityObject is extended with $<<$ ADEElement $>>$. Since Terrains@CityGML is an extension of CityGML, therefore defining the Terrains@-
CityGML classes as subclasses of CityGML and adding the new properties to these subclasses is justified. Also, the method of inheritance with classes and subclasses is easily understandable to people with basic knowledge of UML.

\section{DATASETS AND PROTOTYPE IMPLEMENTATION RESULTS}

The dataset utilised for testing the implementation is 3DTOP10NL (Kadaster. 2015). We used two different tiles (Tile\# 37EN/1 and Tile\# $26 \mathrm{CN} / 2$ each of size $5 \mathrm{~km} \mathrm{X} 6.25 \mathrm{~km}$ ), and 3DTOP10NL with 1368 tiles. The layer of importance is terreinVlak_3D_LODO that contains the DTM of the Netherlands. The details of the input datasets are given in Table 1 notice that each feature of the 2D dataset (polygons representing a road, the terrain, a forest, etc) is triangulated.

To view the structure of Terrains@CityGML representation, a prototype is built. Currently, it permits the user to read a Geodatabase file (*.GDB, the formal in which $3 \mathrm{DTOP} 10 \mathrm{NL}$ is currently available) and outputs the terrain to a Terrains@CityGML representation. The prototype reads the GDB file and removes the redundant vertex information and maps the linear ring structure of triangles to an index based referencing scheme. The results of the prototype testing are given in Table 1 The proposed extension Terrains@CityGML is expected to have the following advantages over current CityGML as shown in Table 2

\section{DISCUSSION AND FUTURE WORK}

With the ever increasing size of 3D terrains, the aim is to develop a compact representation for their efficient storage. What we have proposed in this paper seems rather simple from theoritical point of view but yet it allows to store the terrains in CityGML/GML with around 25 times less storage than the current CityGML solution. The compression factor of 25 is important because it is achieved just only with the removal of gml: LinearRing and inclusion of by-referencing scheme for vertices and triangles. At present, the ADE addresses the issues of huge data volumes and introduces the indexing scheme for vertices and triangles. But 


\begin{tabular}{llrrrrr}
\hline Dataset & layer & \# features & \# triangles & CityGML & Terrains@CityGML & compression factor \\
\hline Tile \#26CN/2 & terrain & 2,753 & $1,162,467$ & $672.7 \mathrm{MB}$ & $25.2 \mathrm{MB}$ & 26.6 \\
Tile \#37EN/2 & terrain & 4,317 & $1,503,241$ & $893.3 \mathrm{MB}$ & $34.9 \mathrm{MB}$ & 25.59 \\
whole 3DTOP10NL & terrain & $2,465,626$ & $1,156,641,666$ & $686.9 \mathrm{~GB}$ & $28.6 \mathrm{~GB}$ & 24.01 \\
\hline
\end{tabular}

Table 1: CityGML and Terrains@CityGML Outputs

\begin{tabular}{lcc}
\hline Criteria & CityGML & Terrains@ CityGML \\
\hline Size Reduction & $\mathbf{X}$ & $\checkmark$ \\
Data Redundancy & $\checkmark$ & $\mathbf{X}$ \\
Topology & $\mathbf{X}$ & $\checkmark$ \\
Semantics & $\checkmark$ & $\checkmark$ \\
Vertex Referencing & $\mathbf{X}$ & $\checkmark$ \\
Triangle Referencing & $\mathbf{X}$ & $\checkmark$ \\
Triangle Encoding & $\mathbf{X}$ & $\checkmark$ \\
Terrain LODs & $\mathbf{X}$ & $\checkmark$ \\
Vertical Triangles & $\mathbf{X}$ & $\checkmark$ \\
Tiling & $\mathbf{X}$ & $\checkmark$ \\
Web rendering & $\mathbf{X}$ & $\checkmark$ \\
\hline
\end{tabular}

Table 2: CityGML vs Terrains@CityGML

we are also working for the remaining problems as highlighed in Section 3 including the concept of LODs for terrain, handling vertical triangles, tiling CityGML datasets, integrating TINs and raster grids, etc. The LOD feature is an important characteristic of 3D city models. It enables gradual refinement of model geometry supplemented with required semantics. Different LODs serve different applications and provide quality information of models. CityGML supports five LODs which in theory can be applied to all the urban features represented with CityGML e.g.buildings, vegetation, bridges, relief, etc. But in reality the concept is not consistent for all the available features. The CityGML LOD concept was primarily defined for the Building module. It describes good enough how a building can be represented in 5 different LODs starting from a footprint (LOD-0) to an architecturally rich build ing with interiors (LOD-4). But this cannot be simply extended to other modules of CityGML e.g.relif, landuse, etc. The description of LODs for other modules is unclear from the perspectives of geometry and semantics. For instance, in case of Relief module, there is no explanation regarding the simplification of a terrain for each LOD. There is no widely-accepted LOD paradigm for terrains in 3D city modelling. We propose to develop a LOD concept exclusively for terrains. At present several criteria in the field of GIS and computer grahics which can serve as a starting point for modelling terrain LODs are being explored like application/context, semantics, distance, size, view focus, etc. As 3DTOP10NL is a $2.8 \mathrm{D}$ model with vertical walls and with no clear mechanism in CityGML to handle vertical walls, we plan to introduce a new class in the schema for identifying such structures. This will be helpful in performing geometrically valid transformations from $3 \mathrm{D}$ to $2 \mathrm{D}$. To alleviate the bottlenecks associated with rendering massive CityGML terrains over web, we also aim to introduce the structures of the concepts of tristrips (Speckmann and Snoeyink, 2001) and stars (Ledoux 2015) as encoding in the CityGML/GML schema. With consecutive ordering of vertices of triangles in the CityGML/GML instances, after the first three vertices forming the first triangle, rendering of each next triangle will only require the next vertex in order. Future work intends to include the other remaining concepts as listed in Table 3 and develop a workable zipped format for Terrains@CityGML. It would be interesting to see the processing and storage of massive datasets with complete
Terrains@CityGML.

\section{ACKNOWLEDGEMENTS}

This research is supported by the Dutch Technology Foundation STW, which is part of the Netherlands Organisation for Scientific Research (NWO), and which is partly funded by the Ministry of Economic Affairs.

\section{References}

Biljecki, F., Stoter, J., Ledoux, H., Zlatanova, S. and Çöltekin, A., 2015. Applications of 3D City Models: State of the Art Review. ISPRS International Journal of Geo-Information 4(4), pp. 2842-2889.

Brink, L., Stoter, J. and Zlatanova, S., 2013. UML-based approach to developing a CityGML Application Domain Extension. Transactions in GIS 17(6), pp. 920-942.

de Laat, R. and Van Berlo, L., 2011. Integration of BIM and GIS: The development of the CityGML GeoBIM extension. In: Advances in 3D geo-information sciences, Springer, pp. 211225.

Dyken, C. and Floater, M. S., 2006. Preferred directions for resolving the non-uniqueness of Delaunay triangulations. Computational Geometry 34(2), pp. 96-101.

Dyn, N., Levin, D. and Rippa, S., 1990. Data dependent triangulations for piecewise linear interpolation. IMA journal of numerical analysis 10(1), pp. 137-154.

Edelsbrunner, H. and Mücke, E. P., 1990. Simulation of Simplicity: A technique to cope with degenerate cases in geometric algorithms. ACM Transactions on Graphics 9(1), pp. 66-104.

Elberink, S. O., Stoter, J., Ledoux, H. and Commandeur, T., 2013. Generation and dissemination of a national virtual 3D city and landscape model for the Netherlands. Photogrammetric engineering \& remote sensing 79(2), pp. 147-158.

Gröger, G. and Löwner, M.-O., 2016. Proposal for a new LoD concept for CityGML 3.0. Technical report, CityGML OGC Work Package 03. 
Gröger, G. and Plümer, L., 2005. How to get 3-D for the price of 2-D? Topology and consistency of 3-D urban GIS. Geoinformatica 9(2), pp. 139-158.

Groger, G., Kolbe, T. H., Nagel, C. and Häfele, K.-H., 2012. OGC City Geography Markup Language (CityGML) Encoding Standard version 2.0.0.

Isenburg, M., Liu, Y., Shewchuk, J. and Snoeyink, J., 2006. Streaming computation of delaunay triangulations. In: ACM Transactions on Graphics (TOG), Vol. 25number 3, ACM, pp. 1049-1056.

Kadaster, 2015. 3DTOP10NL. http://arcg.is/1GKYy7E (Last accessed: April 15, 2016).

Kim, Y., Kang, H. and Lee, J., 2014. Developing CityGML Indoor ADE to manage indoor facilities. In: Innovations in 3D Geo-information sciences, Springer, pp. 243-265.

Kumar, K., Ledoux, H. and Stoter, J., 2016. Comparative analysis of data structures for storing massive TINs in a DBMS. International Archives of the Photogrammetry, Remote Sensing and Spatial Information Sciences XLI-B2, pp. 123-130.

Kumler, M., 1994. An intensive comparison of Triangulated Irregular Networks (TINs) and digital elevation models (dems). Cartographica (2), pp. 1-99.

Ledoux, H., 2008. FieldGML: An alternative representation for fields. In: Headway in Spatial Data Handling, Springer, pp. 385-400.

Ledoux, H., 2015. Storing and analysing massive TINs in a DBMS with a star-based data structure. Technical Report 2015.01, 3D geoinformation, Delft University of Technology, Delft, the Netherlands.

Löwner, M.-O., Benner, J., Gröger, G. and Häfele, K.-H., 2013. New concepts for structuring 3D city models - An extended level of detail concept for CityGML buildings. In: Computational Science and Its Applications-ICCSA 2013, Springer, pp. 466-480.

Nativi, S., Caron, J., Davis, E. and Domenico, B., 2005. Design and implementation of netCDF markup language (NcML) and its GML-based extension (NcML-GML). Computers \& Geosciences 31(9), pp. 1104-1118.

Nouvel, R., Bahu, J.-M., Kaden, R., Kaempf, J., Cipriano, P., Lauster, M. and Casper, E., 2015. Development of the CityGML Application Domain Extension Energy for urban energy simulation. Proceedings of Building Simulation 2015.
OGC, 2011. OpenGIS $®$ Implementation Specification for Geographic Information-Simple feature access-Part 1: Common architecture. OGC document version 06-103r4.

OGC, 2012. OGC city geography markup language (CityGML) encoding standard. Open Geospatial Consortium inc. Document 12-019, version 2.0.0.

Okabe, A., Boots, B., Sugihara, K. and Chiu, S. N., 2009. Spatial tessellations: concepts and applications of Voronoi diagrams. Vol. 501, John Wiley \& Sons.

Rippa, S., 1990. Minimal roughness property of the delaunay triangulation. Computer Aided Geometric Design 7(6), pp. 489497.

Sen, M. and Duffy, T., 2005. GeoSciML: Development of a generic geoscience markup language. Computers \& Geosciences 31(9), pp. 1095-1103.

Shewchuk, J. R., 1996. Triangle: Engineering a 2D Quality Mesh Generator and Delaunay Triangulator. Applied Computational Geometry: Towards Geometric Engineering 1148, pp. 203 222.

Shewchuk, J. R., 1997. Delaunay Refinement Mesh Generation. $\mathrm{PhD}$ thesis, School of Computer Science, Carnegie Mellon University, Pittsburg, USA.

Speckmann, B. and Snoeyink, J., 2001. Easy triangle strips for TIN terrain models. International journal of geographical information science 15(4), pp. 379-386.

Stadler, A. and Kolbe, T. H., 2007. Spatio-semantic coherence in the integration of 3D city models. In: Proceedings of the 5th International Symposium on Spatial Data Quality, Enschede.

van den Brink, L., Stoter, J. and Zlatanova, S., 2013. Establishing a national standard for 3D topographic data compliant to CityGML. International Journal of Geographical Information Science 27(1), pp. 92-113.

VTP, 2012. ITF Format - Virtual Terrain Project. http: //vterrain.org/Implementation/Formats/ITF.html (Last accessed: July 25, 2016).

Woolf, A., Lawrence, B., Lowry, R., Kleese van Dam, K., Cramer, R., Gutierrez, M., Kondapalli, S., Latham, S., Lowe, D., O'Neill, K. et al., 2006. Data integration with the climate science modelling language. Advances in Geosciences 8(8), pp. 83-90. 